\title{
Hard and Soft Multilayered SiCN Nanocoatings with High Hardness and Toughness
}

\author{
S. K. Mishra, ${ }^{1}$ Devraj Verma, ${ }^{1}$ S. Bysakh, ${ }^{2}$ and L. C. Pathak ${ }^{1}$ \\ ${ }^{1}$ CSIR-National Metallurgical Laboratory, Jamshedpur 831007, India \\ ${ }^{2}$ CSIR-Central Glass and Ceramic Research Institute, Kolkota 700032, India \\ Correspondence should be addressed to S. K. Mishra; skm_smp@yahoo.co.in
}

Received 16 September 2013; Revised 6 December 2013; Accepted 9 December 2013

Academic Editor: Qi Ding

Copyright (c) 2013 S. K. Mishra et al. This is an open access article distributed under the Creative Commons Attribution License, which permits unrestricted use, distribution, and reproduction in any medium, provided the original work is properly cited.

\begin{abstract}
Alternate hard and soft layers increase deformation accommodation as thin hard layers slide relative to each other due to shear deformation of low modulus layers. However, the processing of such multilayers is challenging. In the present paper the alternating soft and hard multilayered SiCN coating deposited by magnetron sputtering has been studied and presented. A hardness and modulus of $37 \mathrm{GPa}$ and $317 \mathrm{GPa}$ with elastic recovery of $62 \%$ are achieved by alternate hard and soft layer of Si-C-N by magnetron sputtering. The trilayer films sustained even $2000 \mathrm{gf}$ under indentation without failure though substrate plastically deformed. The fracture toughness value $K_{\mathrm{IC}}$ was measured to be $9.5-10 \mathrm{MPa} \mathrm{m}^{1 / 2}$, significantly higher than many reported hard coatings.
\end{abstract}

\section{Introduction}

For engineering applications, hardness must be complimented with high toughness, which is a property of equal importance as hardness. Toughness is an important mechanical property related to materials resistance against the formation of cracks resulting from stress accumulation in the vicinity of the structural imperfections. In an energetic context, toughness is the ability of the material to absorb energy during deformation until fracture. Therefore, a high toughness coating has high resistance to the formation of cracks under stress and high energy absorbance to hinder crack propagation, whereby preventing chipping, flaking, or catastrophic failure. When the applied stress exceeds the critical value for a particular crack size, there is a growth in the microcracks present which results in brittle failure of the materials as per Griffith's theory [1]. Hard coatings are very important for different industrial application for protection against wear. However hardness must be complimented with good toughness for prolonged performances of these coatings. The nanocrystalline-amorphous composite coatings result in improvement in strength, due to restriction in the dislocation movement for crystallites of very small dimensions which gets pinned when it is surrounded by amorphous matrix.
The amorphous matrix present in the surrounding also helps in reducing problems associated with lattice misfit as the dislocation which might be in the crystalline cannot propagate in to the amorphous phase and vice versa. Secondly, if both the nanocrystalline phase and the amorphous phase are strong, then they have higher cohesive energies [2-4]. The usual mechanism of deformation and mechanical failure is absent or hindered as no dislocation multiplication source can operate and the energy of dislocation in such small crystallites being too high is repelled towards the grain boundaries and annihilates there during growth. However, the crack growth is hindered in nanocrystalline composites and the sizes of the cracks are limited to $2-4 \mathrm{~nm}$. Therefore, nanocomposites coatings also provide high toughness and low friction apart from hardness at the same time [5].

$\mathrm{Si}-\mathrm{C}-\mathrm{N}$ nanocomposites due to their exceptional combination of properties are very promising material for applications as wear and oxidation resistance, optoelectronic material in hostile environment. $\mathrm{Si}-\mathrm{C}-\mathrm{N}$ coatings are desired for many industrial applications such as turbine engines, blades, and wear resistant coatings for automotive industry to enhance the life as well as the performances of the components. They also find application in MEMS device fabrication in the form of field emission displays. 
TABLE 1: Deposition parameters for the single-layer and multilayer Si-C-N films deposited by DC magnetron sputtering.

\begin{tabular}{lcccc}
\hline Type of coating & Nature of coating & Nitrogen pressure (mbar) & Argon pressure (mbar) & Deposition pressure (mbar) \\
\hline Single-layer SiCN coating (A) & Hard & 0.001 & 0.009 & $0.01-0.02$ \\
Single-layer SiCN coating (B) & Soft & 0.099 & 0.001 & $0.09-0.1$ \\
Double-layer SiCN coating (C) (AB) & Hard & 0.001 & 0.009 & 0.01 \\
& Soft & 0.099 & 0.001 & $0.09-0.1$ \\
Double-layer SiCN coating (D) (BA) & Soft & 0.099 & 0.001 & $0.09-0.1$ \\
& Hard & 0.001 & 0.009 & $0.01-0.02$ \\
Trilayer SiCN (E) (ABA) & Hard & 0.001 & 0.009 & 0.01 \\
& Soft & 0.099 & 0.001 & $0.09-0.1$ \\
& Hard & 0.001 & 0.009 & 0.01 \\
\hline
\end{tabular}

Thin film depositions of $\mathrm{Si}-\mathrm{C}-\mathrm{N}$ have been carried out by plasma and ion-assisted deposition, chemical vapour deposition, magnetron sputtering, microwave, and electron cyclotron resonance plasma enhanced chemical vapour (ECRPECVD), ion implantation, pulsed laser deposition, and rapid thermal chemical vapour deposition (RTCVD). At substrate temperatures below $1000^{\circ} \mathrm{C}$, amorphous $\mathrm{Si}-\mathrm{C}-\mathrm{N}$ films are reported to be deposited, while higher temperatures produced crystalline composite films of $\alpha$ - and $\beta-\mathrm{Si}_{3} \mathrm{~N}_{4}$ and $\alpha$ - and $\beta$-SiC. Many reports are available on single-layer hard coatings of SiCN nanocomposite coatings and hardness and modulus has been tailored by varying the plasma or process parameter during deposition and films with less hardness to high harness more than $40 \mathrm{GPa}$ has been achieved.

The limitations associated with monolithic coatings such as lack of multifunctional character and high residual stresses and problems associated with adhesion to substrate have led to increasing use of multilayer coatings. Multilayer coatings not only offer the combination of attractive properties from different materials, but also have observably increased tribological performance over monolithic coatings. The multilayers have advantage that use of one interlayer or several interlayers enhances the adhesion of coatings by reducing the mismatch in mechanical, chemical, or thermal properties between coatings and substrate. Alternating layers can improve the fracture toughness either by introducing layer interfaces to stop cracks or providing a tough medium through which propagation of cracks is prohibited.

A review paper by Stueber et al. [6] presents recent developments in hard, wear resistant thin films based on multilayer coating concept. There are many factors that contribute to enhanced hardness in multilayers.

Multilayers with alternating soft and hard layers increase deformation accommodation as thin hard layers slide relative to each other by virtue of the shear deformation of low modulus layers. However the processing aspect of multilayers is much more demanding with respect to the process control and material selection, number of layers required ensuring sufficient interface quality, stability, and avoiding interfacial reactions between layers that can decrease interfacial strength.

Lot of research is being done on the multilayer coatings alternated with two materials forming the bilayer period [7-9]. The two dissimilar material layers however face the problem of interface stresses, thermal mismatch, and lattice mismatch of the two different materials. There is very little evidence in the literature on the deposition of multilayer coatings formed by depositing the hard and soft coating of the same material. Research has been done on the effect of pressure and nitrogen on the hardness of the $\mathrm{Si}-\mathrm{C}-\mathrm{N}$ coatings [10-17]. The research on different aspect of single-layer hard SiCN coatings has also been reported by one of the authors Mishra et al. in the literature, where it showed very fine nanocrystalline dispersion of size $2-10 \mathrm{~nm}$ in SiCN amorphous matrix $[18,19]$. The films were very hard ranging between 10 and $35 \mathrm{GPa}$ dependent on the deposition parameters.

Deposition pressure and nitrogen to argon ratio can play a dominant role in making a hard film (hardness greater than $20 \mathrm{GPa}$ ) or a soft film (hardness lesser than $20 \mathrm{GPa}$ ). The deposition of alternate hard and soft layers of the same Si-C$\mathrm{N}$ material will circumvent the problems of interface stresses, thermal mismatch, and lattice mismatch of the two different materials soft and hard layers.

In the present paper our research on the microstructural study on the interface of the alternate hard and soft layers of $\mathrm{Si}-\mathrm{C}-\mathrm{N}$ and mechanical and fracture behaviour of hard and tough SiCN multilayered structure deposited by magnetron sputtering is discussed.

\section{Experimental}

Single-layer and multilayer $\mathrm{Si}-\mathrm{C}-\mathrm{N}$ hard and soft coatings were deposited by reactive DC magnetron sputtering on silicon (Si-100) and stainless steel (SS-304) substrates, using single compact target of $\mathrm{SiC}$ in argon-nitrogen atmosphere of plasma. The deposition pressure and nitrogen to argon ratio are varied to generate the comparatively soft (hardness less than $20 \mathrm{GPa}$ ) and hard (hardness more than $30 \mathrm{GPA}$ ) coatings of the same material, that is, $\mathrm{Si}-\mathrm{C}-\mathrm{N}$ and then use them to generate multilayer coating alternated hard and soft coatings. All coatings were deposited at $365^{\circ} \mathrm{C}$ substrate temperatures and at 150 watt power. Each layer was deposited for 2-hour duration. The deposition parameters are given in Table 1.

Microstructural studies were carried out using AFM (SEIKO 400 Japan) and TEM (FEI Tecnai G230ST, The Netherlands). Nanoindentation (XP, MTS/Agilent, USA) experiments were done at varying depths of the film with strain rate of $0.05 \mathrm{~s}^{-1}$. Poisson's ratio was taken as 0.25 for calculations of all parameters. A harmonic displacement of $2 \mathrm{~nm}$ at a frequency of $45 \mathrm{~Hz}$ was initialized for the experiments. 


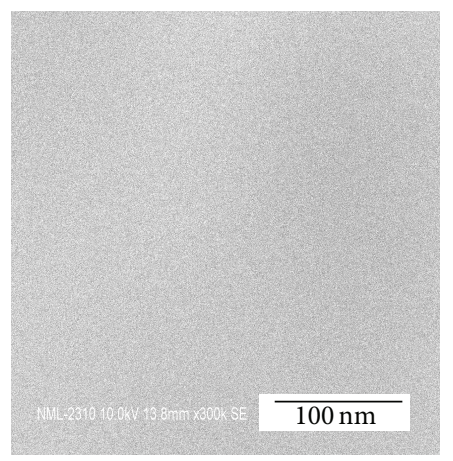

(a)

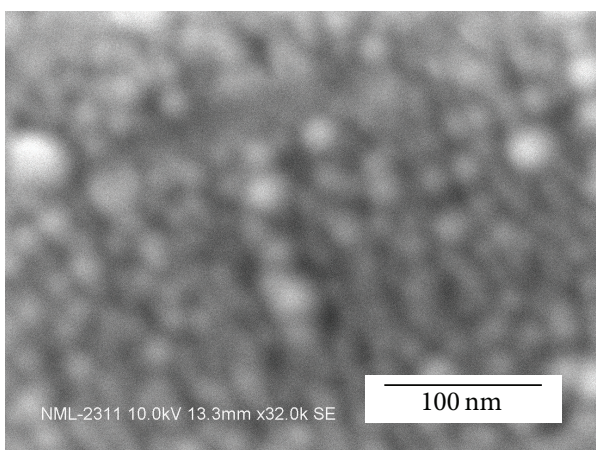

(b)

FIGURE 1: SEM image of SiCN film on SS: (a) single-layer A, hard film and (b) single-layer soft film, B.

The depth for each indentation was fixed as $500 \mathrm{~nm}$ and the experiment was carried out in continuous stiffness mode. The average hardness and modulus for each indent were averaged between the depth of $50 \mathrm{~nm}$ to $500 \mathrm{~nm}$ and the values reported are the average of 8 indents. The TEM samples for interface study were prepared by cutting very thin sample from cross section, then lapped, and finally ion beam thinned for TEM transparency. The percentage elastic recovery of the films was calculated from load-depth curve obtained by nanoindentation and by measuring total area under maximum loading and the retained plastic area with formula given below

$$
\begin{aligned}
& \text { Percentage Elastic Recovery } \\
& =\left\{\frac{(\text { Total area under the curve }- \text { Plastic area })}{\text { Total area }}\right\} * 100 .
\end{aligned}
$$

The microhardness was measured by Leica Germany at $25 \mathrm{gf}$ load. The effect of higher loads was studied by indenting at higher loads of $100 \mathrm{gf}, 200 \mathrm{gf}, 300 \mathrm{gf}, 500 \mathrm{gf}, 1000 \mathrm{gf}$, and $2000 \mathrm{gf}$ on the film. For each film the value presented is average of 6 indents.

\section{Results and Discussion}

The thicknesses of the single-layer hard (A) coating deposited for $2 \mathrm{hrs}$ was about 2.5 micrometer, whereas soft film (B) when deposited for $2 \mathrm{hrs}$ was 1.7 micrometer. Later all the layered films soft or hard were deposited in the desired sequence for $2 \mathrm{hrs}$ each. The sequences were $\mathrm{AB}, \mathrm{BA}$, and ABA.

SEM images of the Si-C-N films deposited at different pressures (films A and B) are given in Figure 1. It was observed that the films deposited at the pressure of $10 \mathrm{~Pa}$ had larger particle sizes (100-200 nm) but in the film deposited at 1 Pa no particles could be resolved by SEM so they were very fine. The microstructure for the film deposited at $1 \mathrm{~Pa}$ showed densely packed which may be responsible for its high hardness value (around $32 \mathrm{GPa}$ ) whereas the microstructure for films deposited at $10 \mathrm{~Pa}$ showed the presence of globular structures with variable dimensions which may be the reason for their comparatively low hardness values (around 9.6 GPa). The introduction of nitrogen in larger ratio (nitrogen argon ratio around 99:1) and increase in deposition pressure (around $10 \mathrm{~Pa}$ ) for film $\mathrm{B}$ is responsible for the significant particle growth, due to lower deposition rates giving enough time to the atoms in terms of mobility and diffusion. The double-layer Film $C(A B)$ the top layer is soft film B had larger size particles $(1 \mu \mathrm{m})$ as seen in SEM image for bilayer.

SFM studies of different single and multilayer sequence were also carried out. The hard film was having very fine particle size in the film in the range of less than $50 \mathrm{~nm}$, whereas the soft film B showed 100-120 nm particle size Figure 2(b). The film $C(A B$, hard-soft) showed its particle size around $50 \mathrm{~nm}$. The double-layer film D soft hard (BA) showed 50$70 \mathrm{~nm}$ growth on surface. The trilayer film ABA had larger growth on top it was found to be around $300 \mathrm{~nm}$. The films were dense as is revealed by AFM. Figures 2(a) and 2(b) presents the AFM images of double-layer film $C(A B)$ and trilayer film E (ABE).

\section{Interface Study of the Trilayer (ABA) SiCN Film}

The interface cross sectional studies of trilayer film $\mathrm{C}$ which was consisting of hard-soft-hard three layers on silicon substrate was studied by Transmission electron microscope. Figure 3(a) shows the interface of Si substrate and the first hard layer. It was observed that they had sharp interface and the individual first hard layer A in the trilayer film was amorphous as confirmed by the SAED of film (inset of Figure 3(a)). The EDX of the film showed mainly the presence of $\mathrm{Si}, \mathrm{N}$, and $\mathrm{C}$. Though the interface between the hard and soft layers was sharp, a very thin $5 \mathrm{~nm}$ reacted zone was observed at the interface between SiCN layer A and the substrate as can be seen in Figure 3(b).

The interface of the layer A, hard film, and the layer B, soft film, is shown in Figure 4(a). Here the interface was sharp and no delamination was observed. A nanocolumnar type growth morphology was observed in the soft film with larger particle sizes compared to hard film A. This was complimentary with the surface microstructure revealed by AFM and SEM. EDS composition analysis of the soft film also showed the presence 


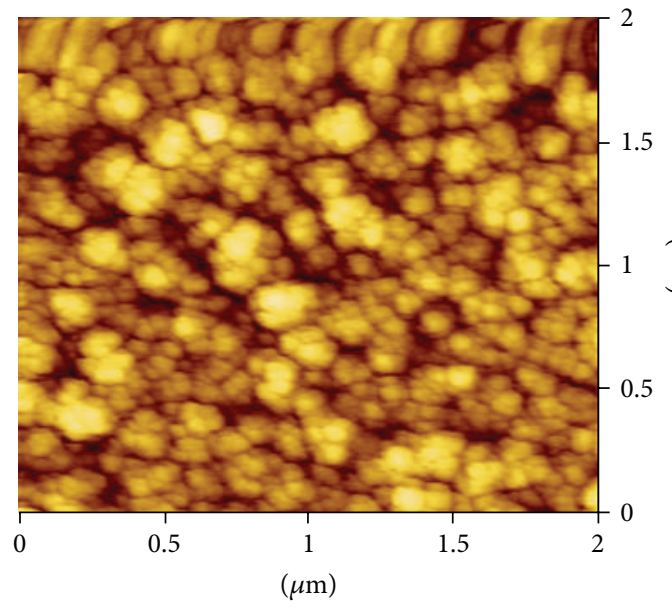

(a)

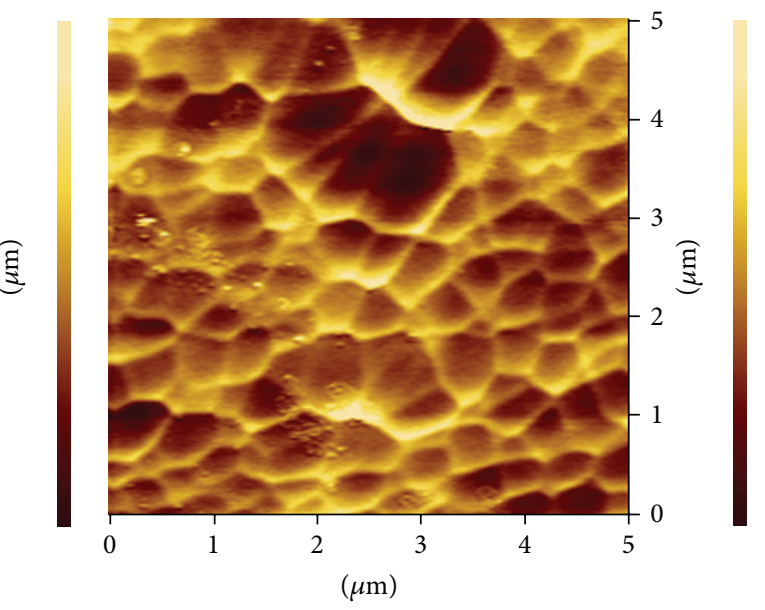

(b)

FigURE 2: AFM topography image of multilayer layer SiCN film on Si: (a) film C (layer AB) and (b) triple layer SiCN film E (ABA).

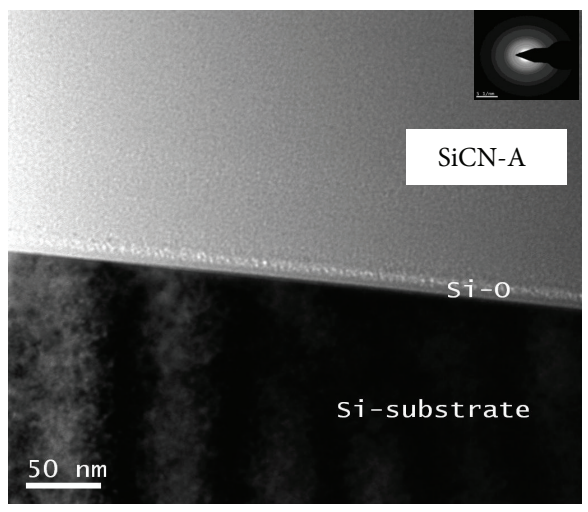

(a)

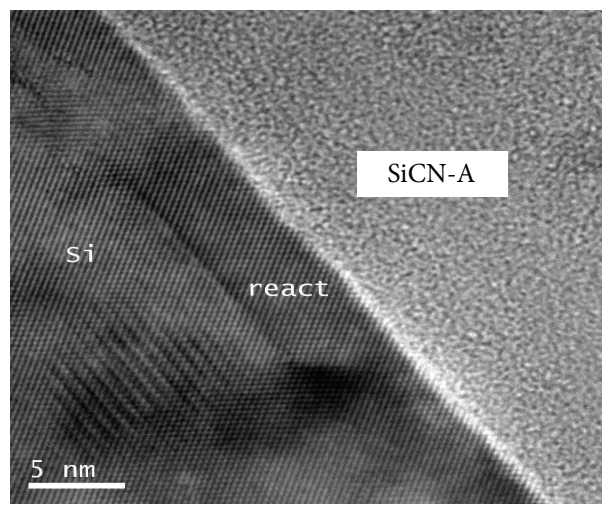

(b)

FIGURE 3: Cross-sectional TEM microstructure: (a) Si and hard SiCN-A interface, SAED of SiCN hard layer A, and (b) high-resolution image of the interface and the reacted zone.

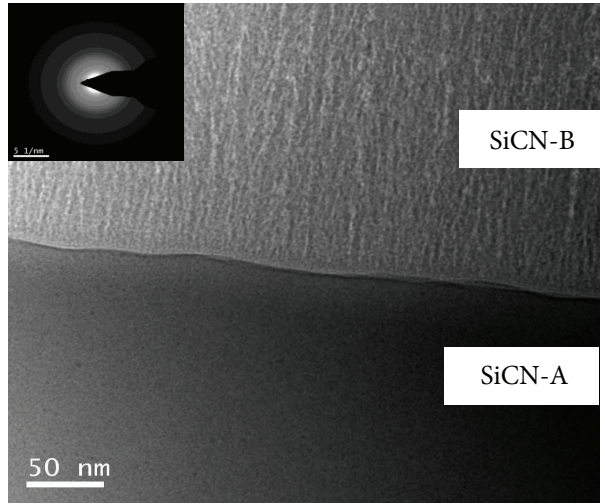

(a)

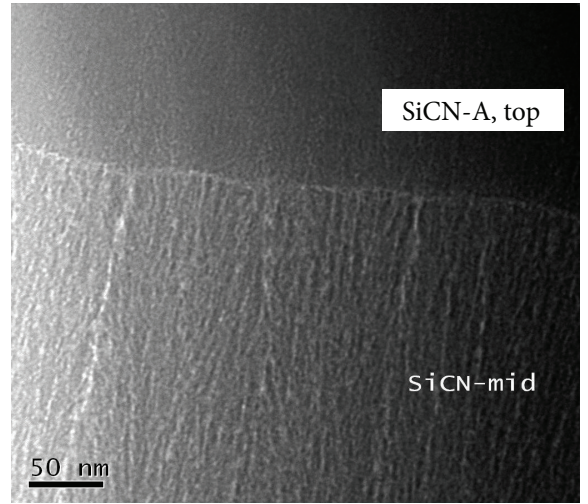

(b)

Figure 4: Cross-sectional TEM microstructure of SiCN layered film: (a) AB layer interface and SAED from Layer B and (b) B middle soft A top hard layer interface. 


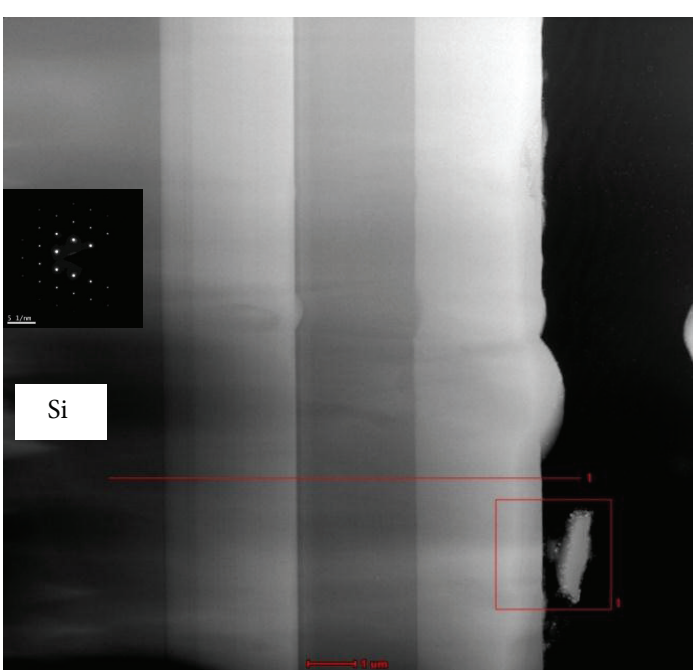

(a)

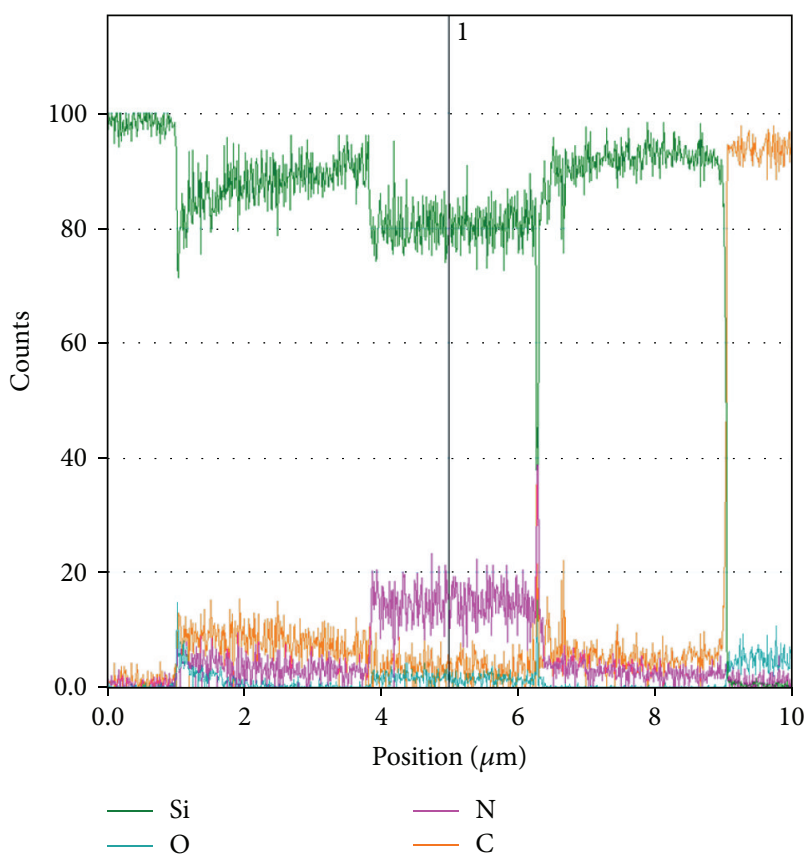

(b)

FIGURE 5: TEM microstructure SiCN film E (a) trilayer ABA, inset SAED of substrate Si, and (b) corresponding elemental EDS mapping across the line of interface shown in (a).

of SiCN mainly; however, the nitrogen was found to be more in case of soft film. It was so expected since the deposition was done at higher nitrogen fraction in the plasma compared to the hard film where nitrogen was much less. Nitrogen incorporation in the film reduces the hardness and has been reported in other systems of film such as TiBN, CN due to the formation of different soft phases consisting of different ratios of $\mathrm{C}$ and $\mathrm{N}$ and Si. The SAED of layer B also essentially showed broad halo ring typical of an amorphous phase SAED (inset of Figure 4(a)). The columnar growth is due to the presence of nitrogen species which leads to slower growth rate and enough mobility of ad atoms leading to more structured growth, whereas in hard layer A the deposition rate was much higher compared to layer B.

Figure 4(b) shows the interface of the middle B layer and the top hard layer. Here it is clear that the columnar grown is visible in B layer only and in A layer the particles are very fine. The interface was found to have sharp boundary, and no other phase was observed in cross section study.

Figure 5(b) shows the nanoprobe STEM-EDS composition line-scan profile across the interface along the line drawn in Figure 5(a), which confirms the sharp boundaries at interface and having higher amount of nitrogen and lesser amount of $\mathrm{Si}$ in soft layer B compared to the hard layers A on its both sides, as was discussed above.

Mechanical properties such as hardness and modulus of the film were studied through nanoindentation using Berkovich indenter. The sample A showed an appreciable hardness $31 \mathrm{GPa}$ with 266.6 GPa modulus. Whereas B was soft having $9 \mathrm{GPa}$ and $115 \mathrm{GPa}$ hardness and modulus. The reason behind high hardness in film $A$ was the formation of $\mathrm{Si}_{3} \mathrm{~N}_{4}$ and $\mathrm{C}_{3} \mathrm{~N}_{4}$ which were found to be absent in the soft film $\mathrm{B}$, as has been reported earlier by one of the present authors [20]. These phases, due to their strong covalent bonds and very fine crystallites in amorphous matrix, impart high hardness to the material. Then, double-layer films were deposited (Film C $(\mathrm{AB})$ and Film D (BA)). These coatings showed increase in hardness as well as increase in Young's modulus and were found to be (hardness: 25.9 and $36.4 \mathrm{GPa}$ ) (modulus: 266, $339 \mathrm{GPa})$, respectively. The hardness of film $\mathrm{C}$ was lesser than the individual hard film $\mathrm{A}$ due to the presence of soft layer and it gives a composite hardness. In the case of film $\mathrm{D}$ however the film hardness and modulus were more than the individual hard film. It is due to the reason that upper most layer is of hard film and underlayer soft layer gives a support and resilience, which in case of single layer was substrate. The thickness of the effective composite film increased; hence, the effect of substrate decreased. Hence higher value trilayer coating Film E (ABA) showed a similar range of hardness and modulus as that of $\mathrm{D}$ but with higher percentage elastic recovery. The underlayer as soft layer seems to increase the hardness and modulus. The values for hardness, modulus, and percentage elastic recovery for different layers are given in Table 2. It was observed that film E with trilayer (ABA) had higher hardness of $36.4 \mathrm{GPa}$ with modulus $317 \mathrm{GPa}$ along with $62 \%$ elastic recovery. This also reveals that trilayer film with ABA combination was tougher.

For fracture behaviour of the different layers microhardness studies were performed on these films. Table 3 shows the hardness obtained for different layers on SS and Si substrates. However, in some cases hardness could not be measured due to the presence of cracks at those loads; hence, values are not 
TABLE 2: Hardness, modulus of elasticity, percentage elastic recovery of single-layer, and multilayer by nanoindentation.

\begin{tabular}{lcccc}
\hline Film & No. of layers (substrate-SS304) & Hardness (GPa) & Modulus of elasticity (GPa) & Percentage elastic recovery (\%) \\
\hline A & 1 & 31.7 & 266.57 & 59.8 \\
B & 1 & 9.629 & 115.745 & 55.0 \\
C (AB) & 2 & 25.978 & 266.138 & 52.6 \\
D (BA) & 2 & 36.43 & 339.387 & 52.3 \\
E (ABA) & 3 & 36.394 & 317.236 & 61.9 \\
\hline
\end{tabular}

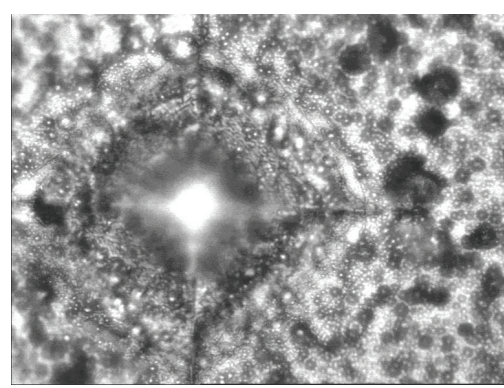

(a)

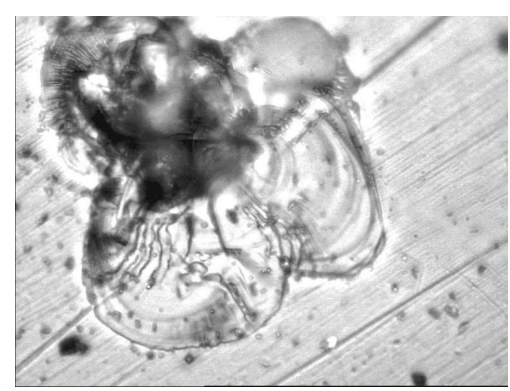

(b)

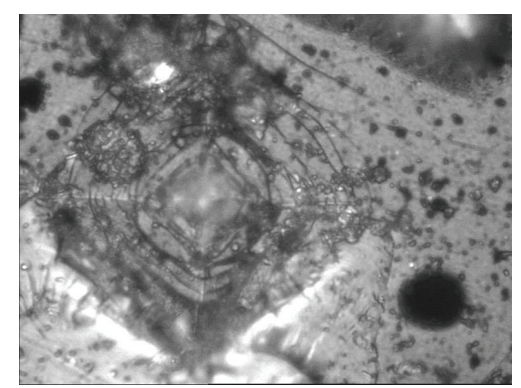

(c)

Figure 6: Indentation picture of SiCN film on SS 304 (a) film E; HSH layer at 2000 gf, (b) Film A at 1000 gf and (c) Film D; SH layer at 1000 gf.

TABLE 3: Hardness values by microhardness tester.

\begin{tabular}{lcccccc}
\hline Load $\rightarrow$ & 25 gf & 100 gf & 200 gf & 300 gf & 500 gf & 1000 gf \\
\hline A (SS 304) & 6630 & 6061 & 4552 & 4102 & - & - \\
B (SS 304) & 3891 & 3181 & 2153 & 1647 & & - \\
C (AB) (SS 304) & 3690 & 3120 & 2850 & 2999 & 3514 & - \\
D (BA) (SS 304) & 4077 & 4091 & 3066 & 3308 & 2875 & - \\
E (ABA) (SS 304) & 5868 & 3713 & 3314 & 2960 & 2768 & 2514 \\
A (Si 100) & 4077 & 4091 & 3066 & 3308 & 2875 & - \\
E (ABA) (Si 100) & 3690 & 3120 & 2850 & 2999 & 3514 & - \\
\hline
\end{tabular}

presented. The values of hardness are different and higher than those from nanoindentation, as in nanoindentation the hardness measured is dynamic and at maximum depth without elastic recovery, whereas in microhardness the hardness values are measured after elastic recoveries have taken place after unloading. Hence, in both the cases hardness values depend on the elastic-plastic behaviour of the composite coatings. The decrease of hardness with load is obvious since with increase of load depth of indentation increases, substrate starts influencing, and composite harness is measured. It is clear from Table 3 that hardness could be measured up to 1000 gf for only trilayer film E.

From fracture behaviour of the films, it was observed that trilayer film had better resistance to loading than individual hard coatings. A soft layer in between played a significant role in building resistant behaviour against indentation. In the fracture in case of film deposited on steel it was observed that the film did not fail even up to $2000 \mathrm{gf}$ but the substrate got plastically deformed resulting in pileups as can be seen in figure lines along the side of the indents but still the film did not show the cracking or spalling (Figure 6(a)). However such failures took at 1000 gf for the film A (Figure 6(b)), spalling is clearly seen. The double-layer film C having hard and soft layer also showed failure at $1000 \mathrm{gf}$, but film D having double layer as soft and hard was able to sustain 1000 gf (Figure 6(c)) though here pileups were more than sample E, so the effect of thickness of the film also plays a role; however, for film $\mathrm{E}$ the structure shown is for $2000 \mathrm{gf}$. It clearly demonstrates that three-layer films were tougher. Hence a soft layer film is helping in increasing the load bearing capacity of the film. In multilayer structure the microcracks tend to branch and deflect at the interface between two alternating layers of different materials. The resulting deflection and branching can lead to reduced stress intensities at the crack tips. This means that at every layer interface the cracking process must start all over again, which takes energy and leads to a tougher coating. At the same time the columnar growth of the soft layer SiCN film beneath or in between hard layers is also added to increase the toughness of the multilayered film due to the stress distribution and deflection. The thickness of the bilayer and trilayer films are also more that will also play role in load bearing capacity of the film. A thicker film will fail at higher loads; however, as it is clear from the microstructure that film has not failed but the substrate pileups were observed, the film remained intact at higher loads also for trilayer film.

The fracture toughness of the trilayer film E (ABA) was measured from film deposited on $\mathrm{Si}$ as crack length could be measured. Figure 7 shows the crack developed at $1000 \mathrm{gf}$ load. The toughness of the brittle materials can be calculated by Antis equation [21]:

$$
K_{c}=\beta \times\left(\frac{E}{H}\right)^{1 / 2} \times \frac{P_{\max }}{a^{3 / 2}},
$$

where $\beta$ is an empirical constant which depends on the geometry of the indenter and the value is 0.032 for cube corner indenter and 0.016 for Berkovich and Vicker's indenter. 


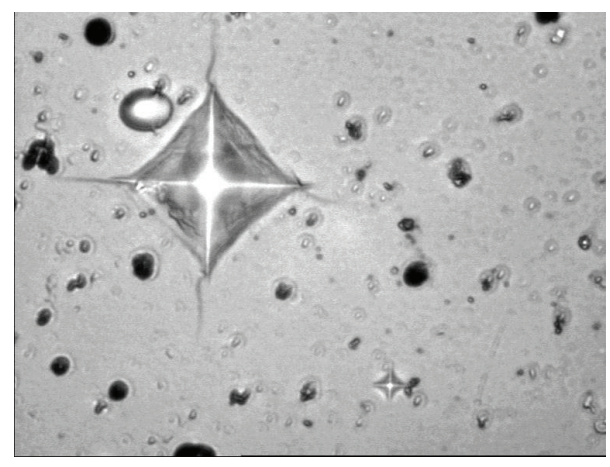

FIGURE 7: A typical cracked surface of SiCN coated multilayer E (ABA) silicon (Si 100) sample at $1000 \mathrm{gf}$ load.

$E$ is the elastic modulus and $H$ is the hardness that was taken from the obtained measured value from nanoindentation as 317.3 and $36.4 \mathrm{GPa}$, respectively. The average crack length was measured from microindentation and was $13.625 \mu \mathrm{m}$. The fracture toughness was calculated at different indents and the average toughness was found to be of the order of 9.5$10 \mathrm{MPa} \mathrm{m}^{1 / 2}$, which is significant higher for a ceramic nanocomposite and single-layer film compared to the reported values. The value of the single hard $\mathrm{SiCN}$ layer was also calculated and was found around 3.5-4 $\mathrm{MPa} \mathrm{m}^{1 / 2}$.

\section{Conclusion}

A detailed microstructure of surface as well the interface has been studied for alternate hard and soft nanocomposite SiCN film deposited by single $\mathrm{SiC}$ target using $\mathrm{RF}$ magnetron sputtering. The average particle size was found to be around $10-30 \mathrm{~nm}$ by AFM and SEM studies for hard film, whereas for soft film it was found to be in the order of 100-150 nm. The layered film showed a mixture of fine grains $80-100 \mathrm{~nm}$. Trilayer film showed particle size around $300 \mathrm{~nm}$. Though the interface was sharp between substrate Si and first hard layer a very thin $5 \mathrm{~nm}$ reacted zone was observed at the interface. Columnar type growth and larger particle sizes compared to hard film were observed in soft film. The elemental composition profile across interface and layers confirms the sharp boundaries at interface between the layers and presence of higher amount of nitrogen and lesser amount of $\mathrm{Si}$ in soft layer B compared to both side hard layers A.

The hardness and modulus for individual soft and hard films were (10 GPa and $115 \mathrm{GPa})$ and $(32 \mathrm{GPa}$ and $266 \mathrm{GPa})$, respectively. The double-layered films hard on soft and soft on hard showed a composite hardness of (26 GPa and $266 \mathrm{GPa}$ ) and (36 GPa and $317 \mathrm{GPa}$ ), respectively. The trilayer film E (ABA) had $37 \mathrm{GPa}$ and $317 \mathrm{GPa}$ hardness and modulus and appreciable elastic recovery of $62 \%$. The fracture behaviour on steel showed that up to $2000 \mathrm{gf}$ force trilayer film E film did not fail but substrate gets deformed resulting in pileups. The crack could be found at $1000 \mathrm{gf}$ for film on Si and fracture toughness value $K_{\text {IC }}$ was measured to be $9.5-10 \mathrm{MPa} \mathrm{m}^{1 / 2}$. The trilayer film E (ABA) showed higher tolerance of the load under the microindentation compared to single-layer or double-layer film. A soft underlayer film below hard layers normally gave a better film in terms of mechanical properties.

\section{References}

[1] J. W. Hutchinson and Z. Suo, "Mixed mode cracking in layered materials," Advances in Applied Mechanics, vol. 29, pp. 63-191, 1991.

[2] M. Gell, "Application opportunities for nanostructured materials and coatings," Materials Science \& Engineering A, vol. 204, no. 1-2, pp. 246-251, 1995.

[3] S. Zhang, H. L. Wang, S.-E. Ong, D. Sun, and X. L. Bui, "Hard yet tough nanocomposite coatings-present status and future trends," Plasma Processes and Polymers, vol. 4, no. 3, pp. 219228, 2007.

[4] A. A. Griffith, "The phenomena of rupture and flows in solids," Philosophical Transactions of the Royal Society A, vol. 221, pp. 163-197, 1921.

[5] S. Vepřek, "Conventional and new approaches towards the design of novel superhard materials," Surface and Coatings Technology, vol. 97, no. 1-3, pp. 15-22, 1997.

[6] M. Stueber, H. Holleck, H. Leiste, K. Seemann, S. Ulrich, and C. Ziebert, "Concepts for the design of advanced nanoscale PVD multilayer protective thin films," Journal of Alloys and Compounds, vol. 483, no. 1-2, pp. 321-333, 2009.

[7] E. G. Wang, "Research on Carbon nitrides," Progress in Materials Science, vol. 41, no. 5, pp. 241-298, 1997.

[8] A. Leyland and A. Matthews, "On the significance of the H/E ratio in wear control: a nanocomposite coating approach to optimised tribological behaviour," Wear, vol. 246, no. 1-2, pp. $1-11,2000$.

[9] J. Ding, Y. Meng, and S. Wen, "Mechanical properties and fracture toughness of multilayer hard coatings using nanoindentation," Thin Solid Films, vol. 371, no. 1, pp. 178-182, 2000.

[10] H. J. Seifert, J. Peng, H. L. Lukas, and F. Aldinger, "Phase equilibria and thermal analysis of Si-C-N ceramics," Journal of Alloys and Compounds, vol. 320, no. 2, pp. 251-261, 2001.

[11] I. Blaszczyk-Lezak, A. M. Wrobel, M. P. M. Kivitorma, I. J. Vayrynen, and A. Tracz, "Silicon carbonitride by remote microwave plasma CVD from organosilicon precursor: growth mechanism and structure of resulting Si:C:N films," Applied Surface Science, vol. 253, no. 17, pp. 7211-7218, 2007.

[12] H. Hoche, D. Allebrandt, M. Bruns, R. Riedel, and C. Fasel, "Relationship of chemical and structural properties with the tribological behavior of sputtered SiCN films," Surface and Coatings Technology, vol. 202, no. 22-23, pp. 5567-5571, 2008.

[13] S. K. Mishra, H. Gaur, P. K. P. Rupa, and L. C. Pathak, "Deposition of nanostructured Si-C-N superhard coatings by rf magnetron sputtering," Journal of Vacuum Science and Technology B, vol. 24, no. 3, pp. 1311-1317, 2006.

[14] A. S. Bhattacharyya, S. K. Mishra, S. Mukherjee, and G. C. Das, "A comparative study of Si-C-N films on different substrates grown by RF magnetron sputtering," Journal of Alloys and Compounds, vol. 478, no. 1-2, pp. 474-478, 2009.

[15] S. K. Mishra, C. Shekhar, P. K. P. Rupa, and L. C. Pathak, "Effect of pressure and substrate temperature on the deposition of nano-structured silicon-carbon-nitride superhard coatings by magnetron sputtering," Thin Solid Films, vol. 515, no. 11, pp. 4738-4744, 2007.

[16] A. Vijayakumar, R. M. Todi, A. P. Warren, and K. B. Sundaram, "Influence of $\mathrm{N}_{2} /$ Ar gas mixture ratio and annealing on optical 
properties of SiCBN thin films prepared by rf sputtering," Diamond and Related Materials, vol. 17, no. 6, pp. 944-948, 2008.

[17] Z.-W. Deng and R. Souda, "XPS studies on silicon carbonitride films prepared by sequential implantation of nitrogen and carbon into silicon," Diamond and Related Materials, vol. 11, no. 9, pp. 1676-1682, 2002.

[18] A. S. Bhattacharyya, S. K. Mishra, and S. Mukherjee, "Correlation of structure and hardness of $\mathrm{rf}$ magnetron sputtered silicon carbonitride films," Journal of Vacuum Science and Technology A, vol. 28, no. 4, pp. 505-509, 2010.

[19] S. K. Mishra and A. S. Bhattacharyya, "Effect of substrate temperature on the adhesion properties of magnetron sputtered nano-composite Si-C-N hard thin films," Materials Letters, vol. 62, no. 3, pp. 398-402, 2008.

[20] A. S. Bhattacharyya and S. K. Mishra, "Raman studies on nanocomposite silicon carbonitride thin film deposited by r.f. magnetron sputtering at different substrate temperatures," Journal of Raman Spectroscopy, vol. 41, no. 10, pp. 1234-1239, 2010.

[21] G. R. Anstis, P. Chantikul, B. R. Lawn, and D. B. Marshall, "A critical evaluation of indentation techniques for measuring fracture toughness: I, direct crack measurements," Journal of the American Ceramic Society, vol. 64, no. 9, pp. 533-538, 1981. 

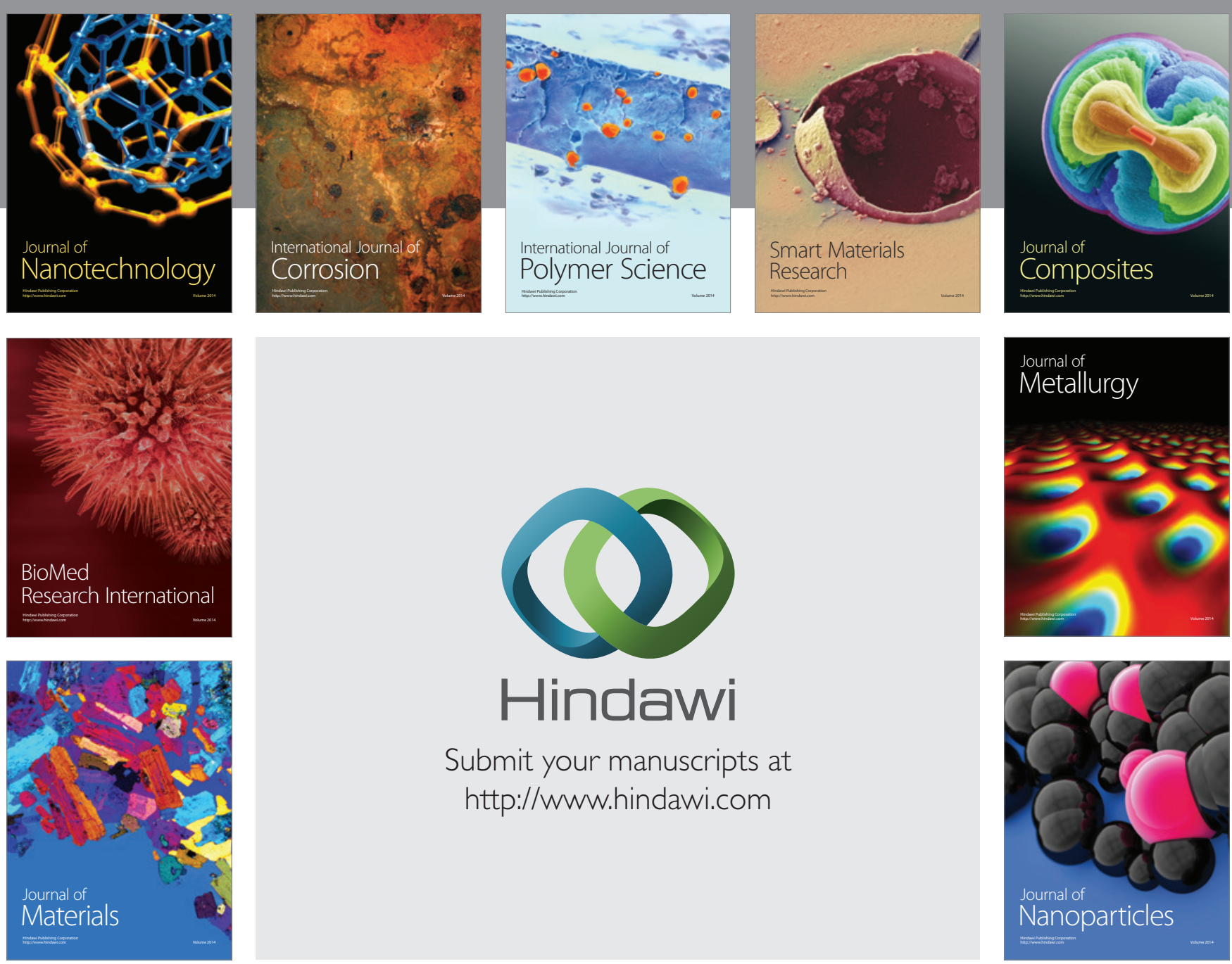

Submit your manuscripts at http://www.hindawi.com
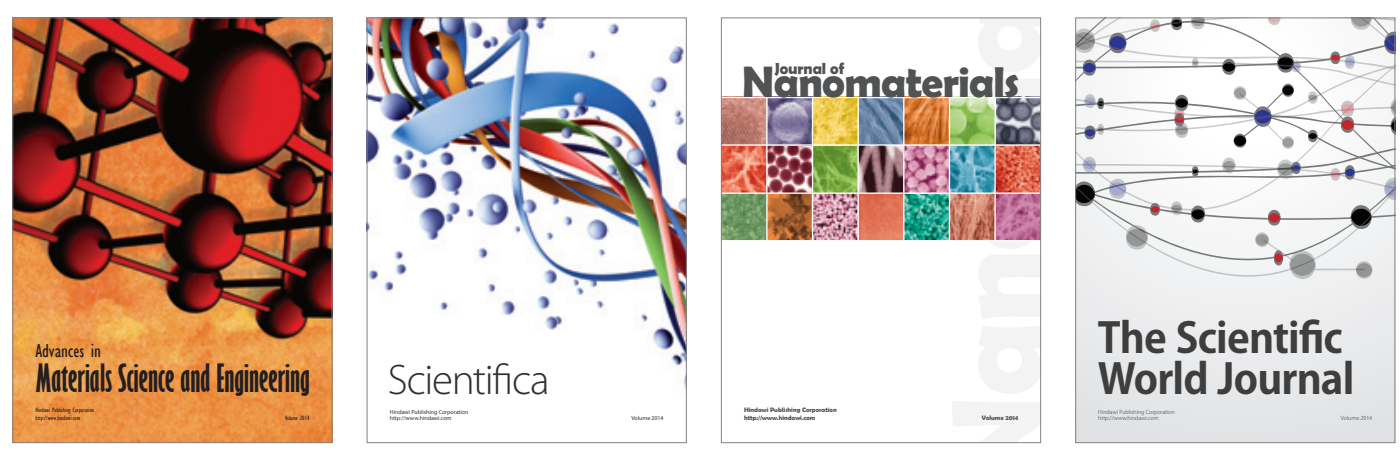

\section{The Scientific World Journal}
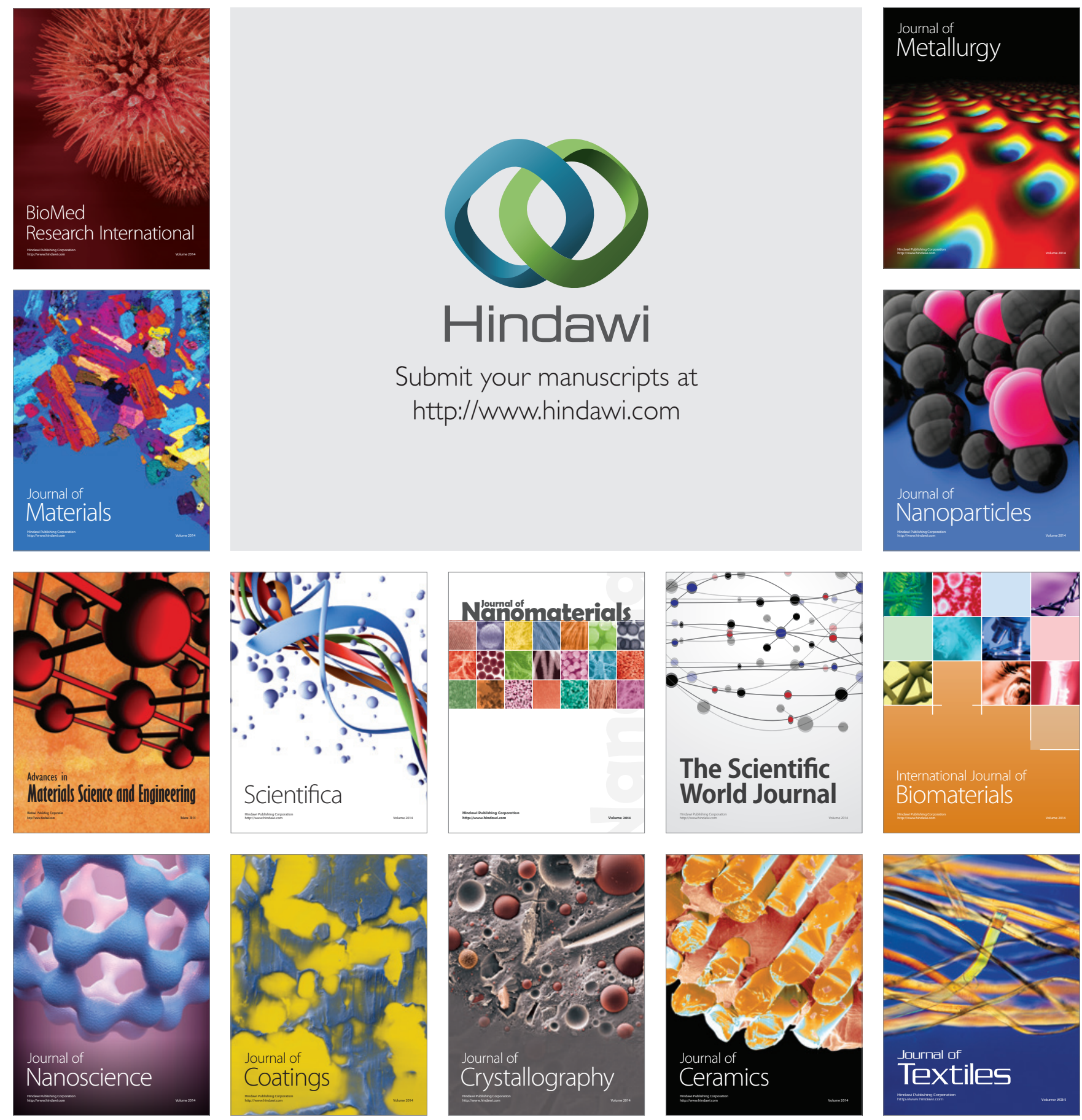